\title{
Metformin Improves Insulin Signaling in Obese Rats via Reduced IKK $\beta$ Action in a Fiber-Type Specific Manner
}

\author{
Benjamin T. Bikman, ${ }^{1,2}$ Donghai Zheng, ${ }^{1,2}$ Daniel A. Kane,, \\ Ethan J. Anderson, ${ }^{1,2}$ Tracey L. Woodlief, ${ }^{1,3}$ Jesse W. Price, ${ }^{4}$ \\ G. Lynis Dohm, ${ }^{1,3}$ P. Darrell Neufer, ${ }^{1,3}$ and Ronald N. Cortright ${ }^{1,3}$ \\ ${ }^{1}$ The Metabolic Institute for the Study of Diabetes and Obesity, East Carolina University, Greenville, NC 27834, USA \\ ${ }^{2}$ Department of Exercise and Sport Science, East Carolina University, Greenville, NC 27834, USA \\ ${ }^{3}$ Department of Physiology, East Carolina University, Greenville, NC 27834, USA \\ ${ }^{4}$ Department of Biology, East Carolina University, Greenville, NC 27834, USA
}

Correspondence should be addressed to Benjamin T. Bikman, benjamin.bikman@duke-nus.edu.sg

Received 26 May 2009; Accepted 27 October 2009

Academic Editor: Eliot Brinton

Copyright (C) 2010 Benjamin T. Bikman et al. This is an open access article distributed under the Creative Commons Attribution License, which permits unrestricted use, distribution, and reproduction in any medium, provided the original work is properly cited.

Metformin is a widely used insulin-sensitizing drug, though its mechanisms are not fully understood. Metformin has been shown to activate AMPK in skeletal muscle; however, its effects on the inhibitor of $\kappa \mathrm{B}$ kinase $\beta$ (IKK $\beta$ ) in this same tissue are unknown. The aim of this study was to (1) determine the ability of metformin to attenuate IKK $\beta$ action, (2) determine whether changes in AMPK activity are associated with changes in IKK $\beta$ action in skeletal muscle, and (3) examine whether changes in AMPK and IKK $\beta$ function are consistent with improved insulin signaling. Lean and obese male Zuckers received either vehicle or metformin by oral gavage daily for four weeks (four groups of eight). Proteins were measured in white gastrocnemius (WG), red gastrocnemius (RG), and soleus. AMPK phosphorylation increased $(P<.05)$ in WG in both lean $(57 \%)$ and obese $(106 \%)$, and this was supported by an increase in phospho-ACC in WG. Further, metformin increased I $\kappa \mathrm{B} \alpha$ levels in both WG (150\%) and RG (67\%) of obese rats, indicative of reduced IKK $\beta$ activity $(P<.05)$, and was associated with reduced IRS1-pSer ${ }^{307}(30 \%)$ in the WG of obese rats $(P<.02)$. From these data we conclude that metformin treatment appears to exert an inhibitory influence on skeletal muscle IKK $\beta$ activity, as evidenced by elevated I $\kappa \mathrm{B} \alpha$ levels and reduced IRS1-Ser ${ }^{307}$ phosphorylation in a fiber-type specific manner.

\section{Introduction}

Insulin resistance is considered a characteristic feature of a clustering of diseases referred to often as the metabolic syndrome [1]. Such associated pathologies include not only cardiovascular disease but also Type II Diabetes Mellitus and, more recently, inflammatory-related metabolic diseases. In this regard, obesity is often associated with insulin resistance, notably in skeletal muscle $[2,3]$. This has immense clinical and economic significance as obesity has reached epidemic proportions not only in the US, but in Western Society at large [4]. Although increased physical activity and nutritional interventions have been used to reduce the progression or reverse obesity (and hence improve insulin action), it has often been met with limited success due to low, long-term patient compliance. In addition, many obese individuals do not posses the physiological capacity for increased physical activity. These circumstances leave pharmacological interventions as an attractive adjunct or alternative therapeutic intervention for treating not only obesity but also associated type II diabetes. Currently, the insulin-sensitizing drug metformin, a member of the biguanide drug class, is widely prescribed. Yet despite its widespread prescription, its basic mechanism of action is poorly understood, slowing the development of even more effective insulin-sensitizing molecules.

The link between obesity and insulin resistance can be partly explained by the NF- $\kappa \mathrm{B}$ pathway and its associated upstream kinases, whose activity is stimulated as a result of the chronic and excessive lipid circulation and accumulation 
in nonadipose tissue sites, a prominent feature of obesity $[5,6]$. Lipid accumulation in skeletal muscle has been shown to be associated with insulin resistance [7], and weight loss appears to reverse this effect $[8,9]$. Ectopic fat deposition can lead to activation of the inhibitor of $\kappa \mathrm{B}$ kinase $\beta$ (IKK $\beta$ ) and induction of its downstream substrate, the inflammatory transcription factor NF- $\kappa$ B [3]. Specifically, $\mathrm{NF}-\kappa \mathrm{B}$ is sequestered in the cytoplasm in an inactive state while complexed with the inhibitor protein called inhibitor $\kappa \mathrm{B} \alpha(\mathrm{I} \kappa \mathrm{B} \alpha)$. Upon stimulation by $\mathrm{IKK} \beta$, which is itself stimulated by both cytokines and lipids [10], $\mathrm{I} \kappa \mathrm{B} \alpha$ is phosphorylated and degraded, resulting in the liberation of $\mathrm{NF}-\kappa \mathrm{B}$, which migrates into the nucleus and activates transcription of inflammatory cytokine genes. Interestingly, in addition to activating NF- $\kappa \mathrm{B}$, IKK $\beta$ has also been shown to directly phosphorylate serine $\mathrm{s}^{312 \text { (Human)/307(rodent) }}$ on insulin receptor substrate (IRS)-1, leading to a decrease in insulin signal transduction [2, 11-13].

In contrast to the insulin-resistant effects of excessive IKK $\beta$ activity, AMP-activated protein kinase (AMPK), a prominent metabolic enzyme, acts to improve insulin sensitivity, potentially counter-acting the deleterious effects of lipid excess. Additionally, many commonly prescribed antidiabetic medications, such as metformin and the thiazolidinediones, are known to increase AMPK activity [14, 15]. In the past few years, research has revealed the role of AMPK as an inhibitor of IKK $\beta$ activity in certain cell types. AMPK has been shown to inhibit both fatty acidand $\mathrm{TNF} \alpha$-induced increases in IKK $\beta$ activity in cultured endothelial cells $[16,17]$, macrophages [18], and astrocytes [19]. Furthermore, metformin treatment has also displayed anti-IKK $\beta$ properties in the liver and has been shown to prevent lipid-induced insulin resistance [20].

Inasmuch as skeletal muscle is the main consumer of glucose in vivo, studying the effects of metformin on IKK $\beta$ activity in skeletal muscle, and the role of AMPK in possibly mediating this response, holds great potential for addressing the obesity-diabetes relationship in skeletal muscle. Metformin has been shown to upregulate AMPK activity in both human and rat skeletal muscle $[15,21]$. Work in endothelial cells and liver has shown that metformin can reduce IKK $\beta$ activity, raising the possibility that activation of AMPK may be a mechanism by which metformin improves insulin sensitivity in skeletal muscle. Therefore, the aim of this study was to (1) determine whether metformin attenuates IKK $\beta$ activity in skeletal muscle of obese rats, (2) determine whether changes in AMPK are associated with reduced IKK $\beta$ activity in skeletal muscle, and (3) examine whether a decrease in IKK $\beta$ activity coincides with reduced phosphorylation of $\mathrm{Ser}^{307}$ on IRS1 in skeletal muscle and whether this translates into increased glucose tolerance.

\section{Materials and Methods}

2.1. Materials. All antibodies used in this investigation were obtained from commercial sources. Antirabbit monoclonal IRS-1 IP antibody for immunoprecipitation was purchased from Santa Cruz Biochtech (Santa Cruz, CA). IRS-1 and Phospho-IRS1 Ser ${ }^{307}$ antibodies were purchased from Millipore (Billerica, MA). Antibodies for phospho-AMPK, AMPK, phospho-ACC, and ACC were purchased from Cell Signaling Technologies (Beverly, MA) and $\mathrm{I} \kappa \mathrm{B} \alpha$ and actin from Santa Cruz Biochtech (Santa Cruz, CA). Horseradish peroxidase-conjugated antirabbit secondary antibodies were purchased from Cell Signaling Technologies (Beverly, MA).

2.2. Animals and Housing. All protocols for animal use were approved by the Animal Care and Use Committee at East Carolina University. Lean and obese male Zucker rats were obtained from Harlan (Indianapolis, IN) and were housed under controlled temperature $\left(23^{\circ} \mathrm{C}\right)$ and lighting (12 hours of light, $0600-1800$ hours; 12 hours of dark; $1800-$ 0600 hours) with free access to water and standard rat chow. Animals were fasted 10 hours before the oral glucose tolerance tests (OGTTs).

\subsection{Metformin Treatment and Oral Glucose Tolerance Testing.} Obese and lean Zucker rats were randomly assigned to receive either control (saline) or metformin $(320 \mathrm{mg} / \mathrm{kg} /$ day $)$ by daily gavage for four weeks ( $N=8$ per group) with the final dose given 4 hours prior to sacrifice. On experimental days, rats were anesthetized with $0.1 \mathrm{~mL} / 100 \mathrm{~g}$ body wt of a mixture containing $90 \mathrm{mg} / \mathrm{mL}$ ketamine and $10 \mathrm{mg} / \mathrm{mL}$ xylazine. With blood flow intact, white gastrocnemius (WG), red gastrocnemius (RG), and soleus muscles were harvested from hind limbs. Samples were rapidly dissected, cleaned, and frozen within seconds in liquid nitrogen and stored at $-80^{\circ} \mathrm{C}$ until analysis.

\subsection{Preparation of Skeletal Muscle Homogenates. Frozen} muscle samples (50-80 mg) were homogenized in ice-cold lysis buffer $50 \mathrm{mM}$ HEPES, $50 \mathrm{mM} \mathrm{Na}^{+}$pyrophosphate, $100 \mathrm{mM} \mathrm{Na}^{+}$fluoride, $10 \mathrm{mM}$ EDTA, $10 \mathrm{mM} \mathrm{Na}^{+}$orthovanadate, $1 \%$ Triton X-100, and protease and phosphatase ( 1 and 2) inhibitor cocktails (Sigma, St. Louis, MO). Homogenates were sonicated for 10 seconds then rotated for 2 hours at $4^{\circ} \mathrm{C}$. After centrifugation for 25 minutes at $14,000 \mathrm{~g}$, supernatants were extracted and protein content was detected using a BCA protein assay (Pierce, Rockford, IL) and individual homogenate volumes were separated into $50 \mu \mathrm{g}$ of protein before being frozen in liquid nitrogen and stored at $-80^{\circ} \mathrm{C}$ until used for immunoblotting.

2.5. Immunoblotting. For IRS-1, homogenates were subjected to $10 \mu \mathrm{L}$ IRS-1 monoclonal IP antibody (Santa Cruz Biotech, Santa Cruz, CA) overnight then coupled to protein A sepharose beads and rotated for 2 hours (Amersham Biosciences, Uppsala Sweden) and eluted with sample buffer. Samples were separated by SDS-PAGE using $7.5 \%$ or $10 \%$ Tris $\cdot \mathrm{HCl}$ gels and then transferred to PVDF membranes for probing by appropriate antibodies. Following incubation with primary antibodies, blots were incubated with appropriate horseradish peroxidase-conjugated secondary antibodies. Horseradish peroxidase activity was assessed with ECL solution (Thermo Scientific, Rockford, IL), and exposed to film. The image was scanned and band densitometry was 


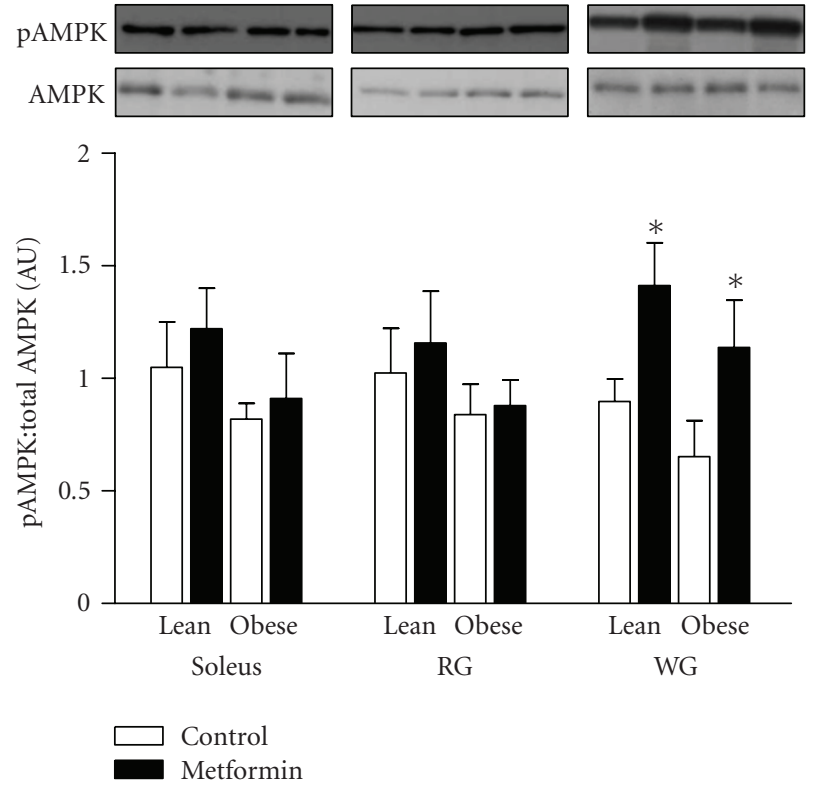

(a)

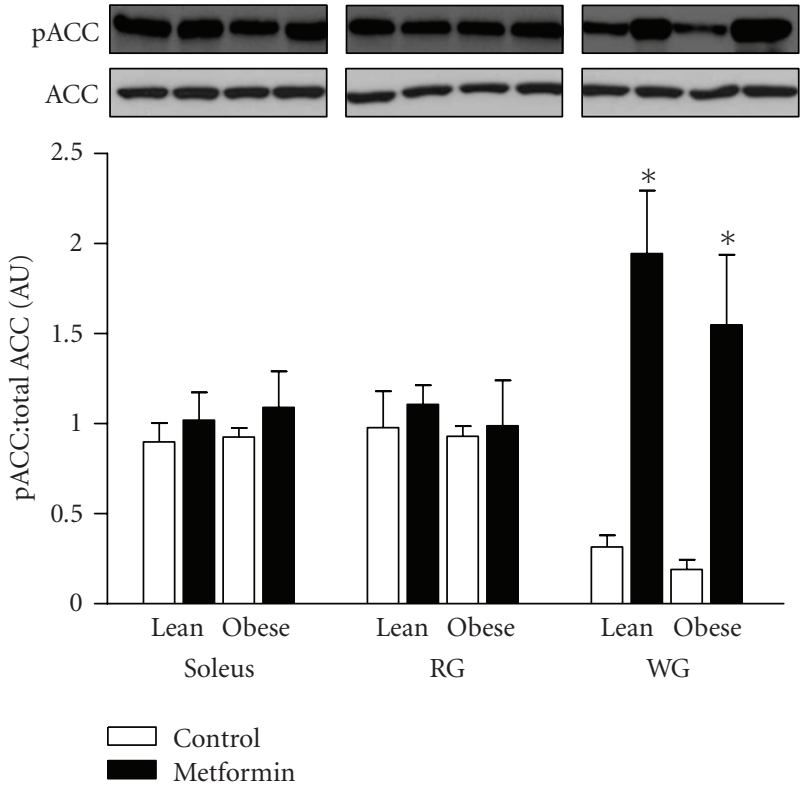

(b)

FIGURE 1: The effect of daily vehicle (open bars) or metformin (filled bars) gavage on AMPK (a) and ACC (b) phosphorylation in soleus, RG, and WG. ${ }^{*} P<.05$. AU: arbitrary units.

assessed with Gel Pro Analyzer software (Media Cybernetics, Silver Spring, MD). Equal loading of proteins was ensured by probing for actin. Content of phospho-proteins (using phospho-specific antibodies) was calculated from the density of the band of the phospho-protein divided by the density of the total protein using the appropriate antibody.

2.6. Statistical Analysis. All data are presented as means \pm SEM. Two-way analysis of variance (ANOVA) was used to compare group and treatment (SPSS, Chicago, IL). Where an interaction was observed, post hoc analysis (Bonferroni and Tukey's HSD) was used to determine significance. Correlation analysis was performed by the Pearson productmoment method. Significance level was established a priori at $P \leq .05$.

\section{Results}

3.1. Metformin and AMPK Activation. AMPK activation was determined by measuring phosphorylation of AMPK and its substrate acetyl-CoA-carboxylase (ACC) while controlling for total levels of both proteins. No difference in AMPK phosphorylation was observed between lean and obese controls in any of the three muscles. Moreover, chronic metformin treatment did not significantly affect AMPK phosphorylation in RG or soleus in neither the lean nor obese rodents (Figure 1(a)), and this was further evident by no change in levels of pACC in these muscles (Figure 2(b)). In contrast, metformin resulted in a significant increase in both phosphorylation of AMPK and ACC in both the lean (57\% for pAMPK and 525\% for pACC) and obese (106\% for pAMPK and $710 \%$ for pACC) rats in WG compared with control animals (Figures $1(\mathrm{a})$ and $1(\mathrm{~b}) ; P<.05)$.

3.2. Metformin and I $\kappa \beta \alpha$. Inasmuch as metformin has been shown to reduce $\operatorname{IKK} \beta$ activity in various tissues, $\mathrm{I} \kappa \mathrm{B} \alpha$ was measured as an indicator of IKK $\beta$ action. Several groups have shown that $\mathrm{I} \kappa \mathrm{B} \alpha$ levels are closely associated with IRS1-Ser phosphorylation and IKK $\beta$ activity [22-24]. No differences in $\mathrm{I} \kappa \mathrm{B} \alpha$ levels were observed between controls animals in either soleus or RG and chronic metformin treatment resulted in no significant effect in soleus from the lean or obese animals (Figure 2(a)). However, I $\kappa \mathrm{B} \alpha$ levels were significantly lower in WG of obese when compared with lean. Further, metformin treatment increased I $\kappa \mathrm{B} \alpha$ levels in both RG and WG of obese animals by $67 \%$ and $150 \%$, respectively, to a level similar to that seen in lean $(P<.05$ for RG, $P<.005$ for WG). When combining all data from WG, $\mathrm{I} \kappa \mathrm{B} \alpha$ showed a strong correlation to AMPK phosphorylation $(r=0.755, P<.005$; Figure 2(b) $)$.

3.3. IRS1-Serine ${ }^{307}$ Phosphorylation. Due to the implicated role of serine phosphorylation of IRS1 in inhibiting insulin signaling, we measured IRS1-pS $\mathrm{S}^{307}$ and controlled for total IRS1. Similar to our observation with $\mathrm{I} \kappa \mathrm{B} \alpha$, there was no significant effect of treatment on soleus $\mathrm{pSer}^{307}$ levels in either lean or obese rodents (Figure 3(a)). Similarly, no differences were observed in RG, although $\mathrm{pSer}^{307}$ in obese tended to be lower in the metformin-treated versus control animals $(P=.061)$. In contrast, $\mathrm{pSer}^{307}$ levels in control animals were significantly higher in obese WG compared with lean $(P<.05)$. Moreover, $\mathrm{pSer}^{307}$ levels were significantly reduced $(30 \%)$ with metformin treatment in WG from obese rodents 


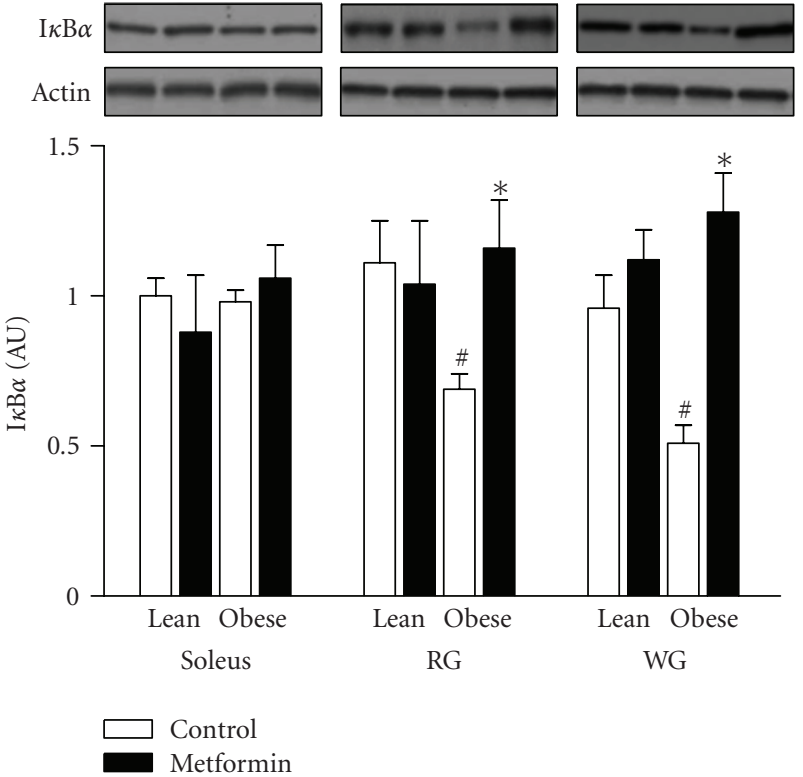

(a)

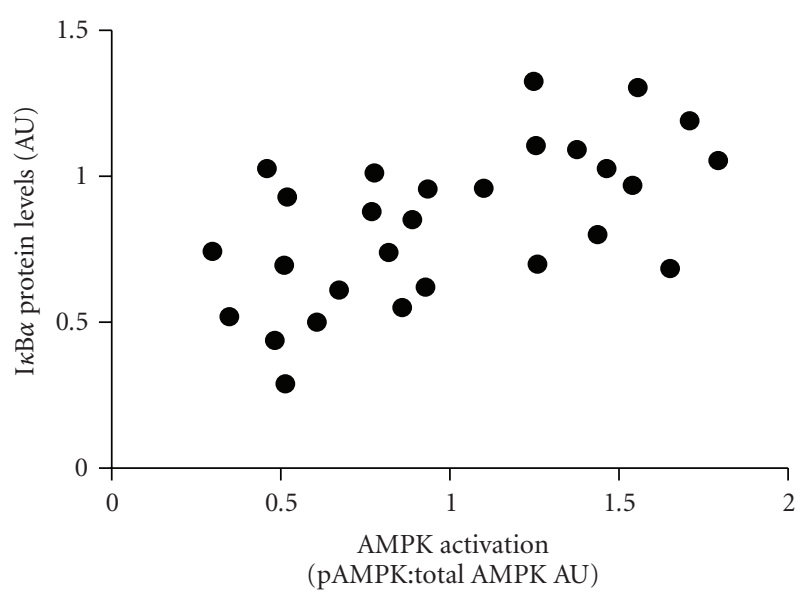

(b)

Figure 2: The effect of vehicle (open bars) or metformin (filled bars) on I $\kappa \mathrm{B} \alpha$ levels in soleus, RG, and WG (a) and the correlation between AMPK and $\mathrm{I} \kappa \mathrm{B} \alpha(\mathrm{b})$ in WG from obese and lean male Zucker rats. Equal loading of proteins was ensured by probing for actin. $P<.05$ for vehicle versus metformin treatment within same phenotype $(*)$ and lean versus obese within a given treatment (\#). AMPK activation and $\mathrm{I} \kappa \mathrm{B} \alpha$ levels were significantly correlated in $\mathrm{WG}(r=0.755, P<.005)$.

$(P<.05)$. Inasmuch as $\mathrm{I} \kappa \mathrm{B} \alpha$ and $\mathrm{pSer}^{307}$ appeared to follow similar trends in WG, we performed a correlation analysis between the two variables. When comparing $\mathrm{pSer}^{307}$ and $\mathrm{I} \kappa \mathrm{B} \alpha$ in all groups, no significant correlation was observed $(r=-0.536, P=.067$; data not shown $)$. However, in WG alone the correlation between $\mathrm{pSer}^{307}$ and $\mathrm{I} \kappa \mathrm{B} \alpha$ reached significance $(r=-0.789, P<.01$; Figure $3(\mathrm{~b}))$, indicating an inverse relationship in WG.

\section{Discussion}

The main finding of the present study is that the beneficial effects of metformin treatment on glucose tolerance in obese, insulin-resistant rodents is associated not only with an elevation in AMPK action, but also $\mathrm{I} \kappa \mathrm{B} \alpha$ levels in WG from obese, insulin-resistant rats. This finding is an indication of reduced IKK $\beta$ activity and suggests that metformin treatment is able to reduce IKK $\beta$ activity and restore $\mathrm{I} \kappa \mathrm{B} \alpha$ protein levels within the muscle. Moreover, these observations are associated with improved insulin signaling, as evidenced by reduced IRS1pSer $^{307}$ levels. Lastly, levels of $\mathrm{I} \kappa \mathrm{B} \alpha$ and IRS1-Ser ${ }^{307}$ were significantly and inversely correlated with metformin-treated obese rats, but only in white muscle, suggesting a fiber-type specific action of metformin on the IKK $\beta$ signaling pathway.

Recent work has supported the role of IKK $\beta$ as an inhibitor of insulin action that results in insulin resistance. In particular, salicylate is known to inhibit the activity of IKK $\beta$, and pretreatment of salicylate in lipid-infused rats rescues glucose tolerance back to similar levels seen in control rats when compared with lipid infusion alone [25]. Further, Yuan et al. [13] showed that $I k k \beta^{+/-}$mice have lower fasting glucose and insulin concentrations compared to $I k k \beta^{+/+}$littermates when on high-fat diets. Additionally, IKK $\beta$ KO mice experience no decrement in insulin sensitivity in response to lipid infusion compared to control mice [26]. Collectively, these findings demonstrate that activation of $\mathrm{IKK} \beta$ is associated with a negative impact on insulin sensitivity. Given these findings and those from other groups demonstrating significant correlations between IKK $\beta$ activity, I $\kappa \mathrm{B} \alpha$ levels, and IRS1-Ser ${ }^{307}$ phosphorylation [9, 22$24]$, we are optimistic that the surrogate outcomes used to determine IKK $\beta$ action, namely, $\mathrm{I} \kappa \mathrm{B} \alpha$ and IRS1-Ser ${ }^{307}$ phosphorylation, IKK $\beta$ 's immediate downstream effectors, adequately reflect IKK $\beta$ action.

In addition to $\operatorname{IKK} \beta$, other lipid-sensitive serine kinases have been implicated in phosphorylating IRS1-Ser ${ }^{312}$. In particular, certain protein kinase C (PKC) isoforms have been shown to be associated with changes in insulin signaling in a similar manner to IKK $\beta$ and some groups present evidence of it being indispensable in potentiating the lipid-induced decay in the insulin signal [23, 27-29]. In particular, in addition to observing a reduction in $\mathrm{I} \kappa \mathrm{B} \alpha$ levels, Itani et al. [23] observed an increase in membrane-associated PKC $\beta$ in the muscle of patients during an acute lipid infusion. However, we found no differences in membrane-associated PKC $\beta$ levels when comparing animals or treatment (data not shown). The discrepancy between these data is possibly due to the diverse conditions and models (e.g., acute lipid infusions versus diet-induced obesity, human versus rodent). In support of our findings, Huang et al. [30] demonstrated that muscle PKC $\beta$ levels did not differ between control and diet-induced obese mice. 


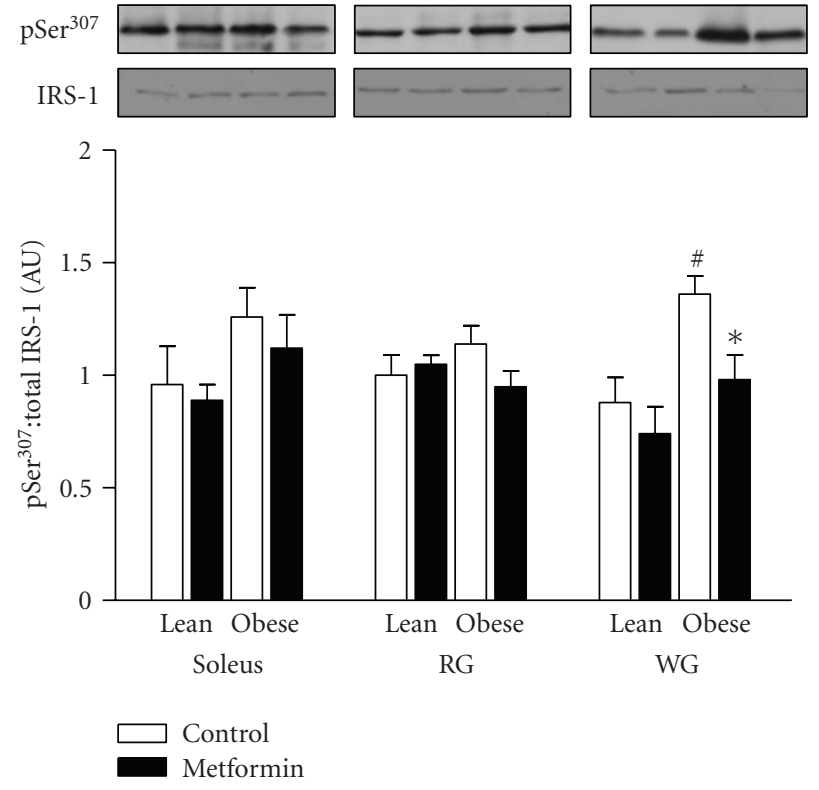

(a)

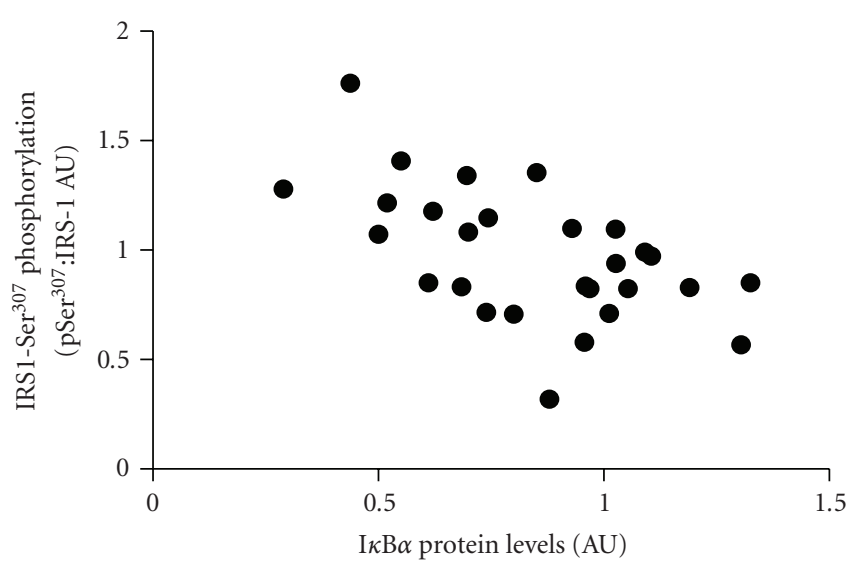

(b)

FIGURE 3: The effect of vehicle (open bars) or metformin (filled bars) on IRS1-pSer ${ }^{307}$ in soleus, RG, and WG (a) and the correlation between $\mathrm{I} \kappa \mathrm{B} \alpha$ and IRS1-pSer ${ }^{307}$ (b) in WG from obese and lean Zucker male rats. $P<.05$ for vehicle versus metformin treatment within same phenotype $(*)$ and lean versus obese within a given treatment $(\#) . \mathrm{I} \kappa \mathrm{B} \alpha$ and IRS1-pSer ${ }^{307}$ levels were significantly related in WG $(r=$ $-0.789, P<.01)$.

Contrary to the reduced insulin sensitivity associated with IKK $\beta$ activity, AMPK plays a key role in improving glucose handling and increasing insulin action. In muscles from sedentary rats, coincubation with insulin and the compound 5-aminoimidasole-4-carboxamide-1- $\beta$ D-ribofuranoside (AICAR; an AMP-mimetic and AMPK activator) has been shown to induce a twofold greater glucose uptake compared to insulin alone [31]. Similarly, AICARperfused rat hindlimbs have been shown to increase glucose uptake compared with controls [32].

Research investigating the novel role of AMPK as a mediator in IKK $\beta$ 's ability to inhibit IRS- 1 function in endothelial cells has revealed that AMPK inhibits both fatty acid- and TNF $\alpha$-induced increases in NF- $\kappa \mathrm{B}$ in cultured endothelial cells [16]. When endothelial cell cultures were incubated with palmitate, inflammatory markers increased, but this response was attenuated in the presence of AICAR. Moreover, AICAR acted to prevent NF- $\kappa \mathrm{B}$ activation in the presence of TNF $\alpha$. This provides strong evidence in support of a role for AMPK in attenuating inflammation and associated cellular metabolic dysfunction. Additionally, metformin, which leads to AMPK activation, dose-dependently inhibits $\mathrm{TNF} \alpha$-induced NF- $\kappa \mathrm{B}$ activation, whereas blocking signaling through AMPK $\alpha 1$ (via small interfering RNA) attenuates metformin- and AICAR-induced inhibition of NF- $\kappa$ B activation by TNF $\alpha$, further supporting a role for AMPK attenuating the inflammation response [17]. A similar relationship was explored in macrophages [33], where treatment of RAW264.7 cells with berberine, a known AMPK activator, was shown to inhibit expression of inflammatory genes including IL- $1 \beta$ and MCP-1 via an AMPK-dependent mechanism.
Research involving metformin has also displayed antiIKK $\beta$ properties in the liver. Cleasby et al. [20] observed elevated levels of $\mathrm{I} \kappa \mathrm{B} \alpha$ in liver from metformin-treated rodents, providing indirect evidence that AMPK may be serving as an IKK $\beta$ inhibitor in an insulin sensitive tissue. These findings provide the rationale for exploring the potential role of AMPK as an IKK $\beta$ inhibitor in skeletal muscle inasmuch as muscle represents the main site of insulin-dependent glucose uptake.

The idea of AMPK attenuating inflammatory activity in skeletal muscle has only recently been investigated and, due to a scarcity of data, a consensus has yet to be reached. For example, Steinberg et al. [34] observed that muscle cells with constitutively active AMPK were protected from $\mathrm{TNF} \alpha$-induced suppression of insulin-stimulated glucose uptake (TNF $\alpha$ has been shown to elicit an increase in IKK $\beta$ activity). In $o b / o b$ mice, it was found that AMPK activity was significantly reduced compared with lean controls and that $\mathrm{TNF} \alpha$ neutralization in $o b / o b$ mice restored AMPK activity to that of lean controls. Furthermore, obese mice exhibited reduced fatty acid oxidation, a defect that was not observed following TNF $\alpha$ neutralization. Lastly, ob/ob mice lacking a functional $\mathrm{TNF} \alpha$ receptor $\left(T N F^{-/-}\right)$enjoy greater insulin sensitivity than control $o b / o b$ mice. It was observed that AMPK activity was higher in obese $T N F^{-/-}$relative to obese controls. Whereas the evidence provided by Steinberg et al. [34] places inflammatory mediators upstream of AMPK, the work by Hattori et al. [17] offers the opposite perspectivethat AMPK inhibits NF- $\kappa$ B pathway activity. Moreover, the observations of the current study extend the work by Hattori et al. [17] in that we provide evidence that AMPK may attenuate IKK $\beta$ action in skeletal muscle. Specifically, we 
observed an increase in AMPK phosphorylation, an increase in $\mathrm{I} \kappa \mathrm{B} \alpha$ levels (suggesting reduced IKK $\beta$ activity), and, finally, reduced levels of IRS1-pSer ${ }^{307}$ in white muscle from metformin-treated obese rats.

In contrast, Ho et al. [35] explored the effects of AICARstimulated AMPK activation in rats in vivo and found no reduction in IKK $\beta$ phosphorylation 60 minutes following an intraperitoneal injection of AICAR despite a robust increase in AMPK activity in skeletal muscle. Further, they did not observe any change in IKK $\beta$ phosphorylation in isolated rat EDL muscles treated with AICAR. These findings appear to indicate that AMPK does not directly regulate IKK $\beta$ activity; however, the acute nature of the study design must be considered. The findings of the current study utilized a chronic treatment intervention and support the possibility that metformin-mediated activation of AMPK attenuates IKK $\beta$ activity, as has been established in hepatic tissue [20].

Interestingly, metformin-induced activation of AMPK occurs in the absence of any changes in ATP/ADP ratio, indicating that a decrease in cellular energy charge is not the link between metformin and AMPK activation [36]. In this regard, synergistic or independent mechanisms should also be considered. For example, metformin has also been shown to partially inhibit mitochondrial complex I and subsequent free radical production [37], raising the possibility that an additional, unique effect of metformin in improving $\mathrm{I} \kappa \mathrm{B} \alpha$ and IRS1-pSer ${ }^{307}$ levels may be related to its effects on mitochondrial function in addition to that of AMPK activation. An association between free radical production, IKK $\beta$ activity, and insulin sensitivity has been established and might prove to be a fruitful area of investigation on this topic.

It is noteworthy that we observed neither an increase in AMPK activity nor a reduction in IKK $\beta$ activity or IRS1$\mathrm{pSer}^{307}$ in soleus with metformin treatment. Similarly, no differences were noted in either AMPK or IRS1-pSer ${ }^{307}$ levels in $\mathrm{RG}$, although $\mathrm{I} \kappa \mathrm{B} \alpha$ levels increased in obese RG with metformin treatment. The singular change in $\mathrm{I} \kappa \mathrm{B} \alpha$ levels without an accompanying reduction in IRS1-Ser ${ }^{307}$ phosphorylation in RG was unexpected, though the comparison of IRS1pSer $^{307}$ levels between metformin-treated and control obese animals did approach statistical significance $(P=.061)$. Future studies seem warranted then to determine if both statistical and physiological significance would be realized in red muscle following a longer treatment time as would be expected in human subjects under chronic metformin prescription.

In contrast to the observations in soleus and RG, AMPK, $\mathrm{I} \kappa \mathrm{B} \alpha$, and IRS1-pSer ${ }^{307}$ levels were all affected by chronic metformin treatment in WG from the obese rats. Specifically, metformin treatment increased AMPK activity and was associated with both an increase in $\mathrm{I} \kappa \mathrm{B} \alpha$ protein levels and a reduction in IRS1-pSer ${ }^{307}$. In accordance with these findings, it should be recognized that other investigators have noted differences in the IKK $\beta$ signaling pathway between muscle fiber types. For example, Bhatt et al. [38] observed a reduction in $\mathrm{I} \kappa \mathrm{B} \alpha$ levels with diet-induced obesity in rat skeletal muscle in a fiber-type dependent manner. Specifically, obesity was associated with decreased levels of $\mathrm{I} \kappa \mathrm{B} \alpha$ in superficial vastus (white, fast twitch-glycolytic), whereas the soleus (red, slow twitch-oxidative) appeared to be protected from such an effect. Additionally, Iglesias et al. [39] observed an AICAR-induced improvement in glucose uptake in white muscle that was not evident red muscle. Inasmuch as metformin may exert its action by altering mitochondrial function, it is possible that given red muscle's prevalence of mitochondria, a greater metformin dose is required to elicit the same response as observed in white muscle.

\section{Conclusion}

In summary, these findings demonstrate that IRS1-Ser ${ }^{307}$ phosphorylation is elevated in certain muscle types of obese, highly insulin resistant Zucker rats. Moreover, metformin treatment is associated with increased AMPK function and $\mathrm{I} \kappa \mathrm{B} \alpha$ levels, at least in white muscle. These findings are novel, in that they offer support for the hypothesis that AMPK may influence IKK $\beta$ action. Indeed, under the experimental conditions present in the current study, AMPK appears to exert an inhibitory effect on IKK $\beta$ in a fiber-type dependent manner, which contributes to the change in muscle insulin signaling. Future studies investigating dose and treatment time for Metformin seem warranted when considering potential effects on the IKK $\beta$ signaling pathway in muscles of red fiber type composition.

\section{Acknowledgments}

This work was supported by Grants R01 046121 (G. L. Dohm), R15 DK061314 (R. N. Cortright), and R01 DK073488 (P. D. Neufer).

\section{References}

[1] S. M. Grundy, H. B. Brewer Jr., J. I. Cleeman, S. C. Smith Jr., and C. Lenfant, "Definition of metabolic syndrome: report of the National Heart, Lung, and Blood Institute/American Heart Association conference on scientific issues related to definition," Circulation, vol. 109, no. 3, pp. 433-438, 2004.

[2] G. Perseghin, K. Petersen, and G. I. Shulman, "Cellular mechanism of insulin resistance: potential links with inflammation," International Journal of Obesity, vol. 27, supplement 3, pp. S6S11, 2003.

[3] M. C. Arkan, A. L. Hevener, F. R. Greten, et al., "IKK- $\beta$ links inflammation to obesity-induced insulin resistance," Nature Medicine, vol. 11, no. 2, pp. 191-198, 2005.

[4] A. H. Mokdad, J. S. Marks, D. F. Stroup, and J. L. Gerberding, "Actual causes of death in the United States, 2000," Journal of the American Medical Association, vol. 291, no. 10, pp. 12381245, 2004.

[5] K. E. Wellen and G. S. Hotamisligil, "Inflammation, stress, and diabetes," Journal of Clinical Investigation, vol. 115, no. 5, pp. 1111-1119, 2005.

[6] B. B. Kahn and J. S. Flier, "Obesity and insulin resistance," Journal of Clinical Investigation, vol. 106, no. 4, pp. 473-481, 2000.

[7] J. D. McGarry, "Banting lecture 2001: dysregulation of fatty acid metabolism in the etiology of type 2 diabetes," Diabetes, vol. 51, no. 1, pp. 7-18, 2002. 
[8] R. E. Gray, C. J. Tanner, W. J. Pories, K. G. MacDonald, and J. A. Houmard, "Effect of weight loss on muscle lipid content in morbidly obese subjects," American Journal of Physiology, vol. 284, no. 4, pp. E726-E732, 2003.

[9] B. T. Bikman, D. Zheng, W. J. Pories, et al., "Mechanism for improved insulin sensitivity after gastric bypass surgery," Journal of Clinical Endocrinology \& Metabolism, vol. 93, no. 12, pp. 4656-4663, 2008.

[10] G. S. Hotamisligil, "Inflammation and metabolic disorders," Nature, vol. 444, no. 7121, pp. 860-867, 2006.

[11] D. Cai, M. Yuan, D. F. Frantz, et al., "Local and systemic insulin resistance resulting from hepatic activation of IKK- $\beta$ and NF$\kappa \mathrm{B}$," Nature Medicine, vol. 11, no. 2, pp. 183-190, 2005.

[12] H. Ghanim, R. Garg, A. Aljada, et al., "Suppression of nuclear factor- $\kappa \mathrm{B}$ and stimulation of inhibitor $\kappa \mathrm{B}$ by troglitazone: evidence for an antiinflammatory effect and a potential antiatherosclerotic effect in the obese," Journal of Clinical Endocrinology \& Metabolism, vol. 86, no. 3, pp. 1306-1312, 2001.

[13] M. Yuan, N. Konstantopoulos, J. Lee, et al., "Reversal of obesity- and diet-induced insulin resistance with salicylates or targeted disruption of IKK $\beta$," Science, vol. 293, no. 5535, pp. 1673-1677, 2001.

[14] S. J. Lessard, Z.-P. Chen, M. J. Watt, et al., "Chronic rosiglitazone treatment restores AMPK $\alpha 2$ activity in insulinresistant rat skeletal muscle," American Journal of Physiology, vol. 290, no. 2, pp. E251-E257, 2006.

[15] M. Suwa, T. Egashira, H. Nakano, H. Sasaki, and S. Kumagai, "Metformin increases the PGC- $1 \alpha$ protein and oxidative enzyme activities possibly via AMPK phosphorylation in skeletal muscle in vivo," Journal of Applied Physiology, vol. 101, no. 6, pp. 1685-1692, 2006.

[16] J. M. Cacicedo, N. Yagihashi, J. F. Keaney Jr., N. B. Ruderman, and Y. Ido, "AMPK inhibits fatty acid-induced increases in NF- $\kappa \mathrm{B}$ transactivation in cultured human umbilical vein endothelial cells," Biochemical and Biophysical Research Communications, vol. 324, no. 4, pp. 1204-1209, 2004.

[17] Y. Hattori, K. Suzuki, S. Hattori, and K. Kasai, "Metformin inhibits cytokine-induced nuclear factor $\kappa \mathrm{B}$ activation via AMP-activated protein kinase activation in vascular endothelial cells," Hypertension, vol. 47, no. 6, pp. 1183-1188, 2006.

[18] B. S. Jhun, Q. Jin, Y. T. Oh, et al., "5-aminoimidazole-4carboxamide riboside suppresses lipopolysaccharide-induced TNF- $\alpha$ production through inhibition of phosphatidylinositol 3-kinase/Akt activation in RAW 264.7 murine macrophages," Biochemical and Biophysical Research Communications, vol. 318, no. 2, pp. 372-380, 2004.

[19] S. Giri, N. Nath, B. Smith, B. Viollet, A. K. Singh, and I. Singh, "5-aminoimidazole-4-carboxamide-1- $\beta$-4-ribofuranoside inhibits proinflammatory response in glial cells: a possible role of AMP-activated protein kinase," Journal of Neuroscience, vol. 24, no. 2, pp. 479-487, 2004.

[20] M. E. Cleasby, N. Dzamko, B. D. Hegarty, G. J. Cooney, E. W. Kraegen, and J.-M. Ye, "Metformin prevents the development of acute lipid-induced insulin resistance in the rat through altered hepatic signaling mechanisms," Diabetes, vol. 53, no. 12, pp. 3258-3266, 2004.

[21] N. Musi, "AMP-activated protein kinase and type 2 diabetes," Current Medicinal Chemistry, vol. 13, no. 5, pp. 583-589, 2006.

[22] E. R. Ropelle, J. R. Pauli, P. O. Prada, et al., "Reversal of diet-induced insulin resistance with a single bout of exercise in the rat: the role of PTP1B and IRS-1 serine phosphorylation," Journal of Physiology, vol. 577, no. 3, pp. 997-1007, 2006.

[23] S. I. Itani, N. B. Ruderman, F. Schmieder, and G. Boden, "Lipid-induced insulin resistance in human muscle is associated with changes in diacylglycerol, protein kinase $\mathrm{C}$, and $\mathrm{I} \kappa \mathrm{B}-$ $\alpha$," Diabetes, vol. 51, no. 7, pp. 2005-2011, 2002.

[24] S. Sinha, G. Perdomo, N. F. Brown, and R. M. O’Doherty, "Fatty acid-induced insulin resistance in L6 myotubes is prevented by inhibition of activation and nuclear localization of nuclear factor $\kappa \mathrm{B}$," Journal of Biological Chemistry, vol. 279, no. 40, pp. 41294-41301, 2004.

[25] M.-J. Yin, Y. Yamamoto, and R. B. Gaynor, "The antiinflammatory agents aspirin and salicylate inhibit the activity of I $\kappa$ B kinase- $\beta$," Nature, vol. 396, no. 6706, pp. 77-80, 1998.

[26] J. K. Kim, Y.-J. Kim, J. J. Fillmore, et al., "Prevention of fatinduced insulin resistance by salicylate," Journal of Clinical Investigation, vol. 108, no. 3, pp. 437-446, 2001.

[27] C. Schmitz-Peiffer, "Protein kinase C and lipid-induced insulin resistance in skeletal muscle," Annals of the New York Academy of Sciences, vol. 967, pp. 146-157, 2002.

[28] G. Frangioudakis, J. G. Burchfield, S. Narasimhan, et al., "Diverse roles for protein kinase $\mathrm{C} \delta$ and protein kinase $\mathrm{C} \varepsilon$ in the generation of high-fat-diet-induced glucose intolerance in mice: regulation of lipogenesis by protein kinase $\mathrm{C} \delta$," Diabetologia, vol. 52, no. 12, 26162620 pages, 2009.

[29] R. Ragheb, G. M. L. Shanab, A. M. Medhat, D. M. Seoudi, K. Adeli, and I. G. Fantus, "Free fatty acid-induced muscle insulin resistance and glucose uptake dysfunction: evidence for PKC activation and oxidative stress-activated signaling pathways," Biochemical and Biophysical Research Communications, vol. 389, no. 2, pp. 211-216, 2009.

[30] W. Huang, R. Bansode, M. Mehta, and K. D. Mehta, "Loss of protein kinase $\mathrm{C} \beta$ function protects mice again diet-induced obesity and development of hepatic steatosis and insulin resistance," Hepatology, vol. 49, no. 5, pp. 1525-1536, 2009.

[31] J. S. Fisher, J. Gao, D.-H. Han, J. O. Holloszy, and L. A. Nolte, "Activation of AMP kinase enhances sensitivity of muscle glucose transport to insulin," American Journal of Physiology, vol. 282, no. 1, pp. E18-E23, 2002.

[32] G. F. Merrill, E. J. Kurth, D. G. Hardie, and W. W. Winder, "AICA riboside increases AMP-activated protein kinase, fatty acid oxidation, and glucose uptake in rat muscle," American Journal of Physiology, vol. 273, no. 6, pp. E1107-E1112, 1997.

[33] H. W. Jeong, K. C. Hsu, J.-W. Lee, et al., "Berberine suppresses proinflammatory responses through AMPK activation in macrophages," American Journal of Physiology, vol. 296, no. 4, pp. E955-E964, 2009.

[34] G. R. Steinberg, B. J. Michell, B. J. W. van Denderen, et al., "Tumor necrosis factor $\alpha$-induced skeletal muscle insulin resistance involves suppression of AMP-kinase signaling," Cell Metabolism, vol. 4, no. 6, pp. 465-474, 2006.

[35] R. C. Ho, M. F. Hirshman, Y. Li, et al., "Regulation of $I \kappa B$ kinase and NF- $\kappa \mathrm{B}$ in contracting adult rat skeletal muscle," American Journal of Physiology, vol. 289, no. 4, pp. C794-C801, 2005.

[36] S. A. Hawley, A. E. Gadalla, G. S. Olsen, and D. G. Hardie, "The antidiabetic drug metformin activates the AMP-activated protein kinase cascade via an adenine nucleotide-independent mechanism," Diabetes, vol. 51, no. 8, pp. 2420-2425, 2002.

[37] M. R. Owen, E. Doran, and A. P. Halestrap, "Evidence that metformin exerts its anti-diabetic effects through inhibition of complex 1 of the mitochondrial respiratory chain," Biochemical Journal, vol. 348, no. 3, pp. 607-614, 2000. 
[38] B. A. Bhatt, J. J. Dube, N. Dedousis, J. A. Reider, and R. M. O'Doherty, "Diet-induced obesity and acute hyperlipidemia reduce $\mathrm{I} \kappa \mathrm{B} \alpha$ levels in rat skeletal muscle in a fiber-type dependent manner," American Journal of Physiology, vol. 290, no. 1, pp. R233-R240, 2006.

[39] M. A. Iglesias, S. M. Furler, G. J. Cooney, E. W. Kraegen, and J.-M. Ye, "AMP-activated protein kinase activation by AICAR increases both muscle fatty acid and glucose uptake in white muscle of insulin-resistant rats in vivo," Diabetes, vol. 53, no. 7, pp. 1649-1654, 2004. 


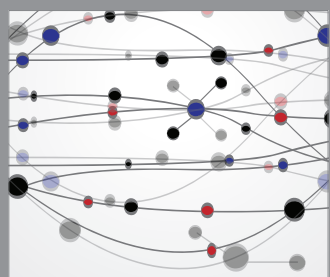

The Scientific World Journal
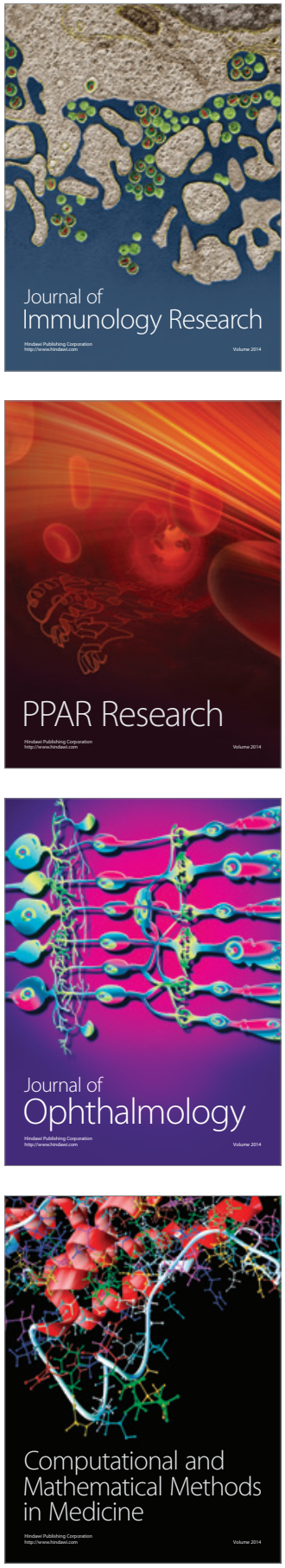

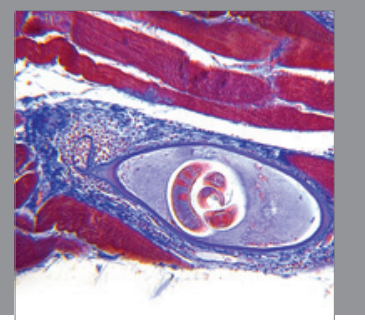

Gastroenterology

Research and Practice
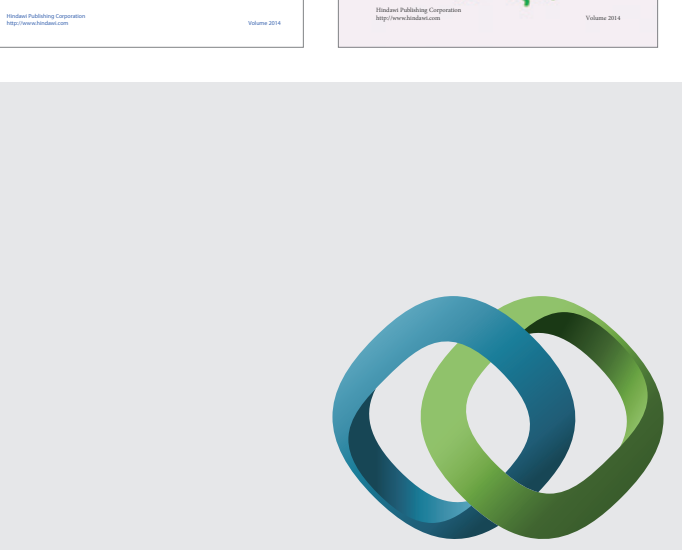

\section{Hindawi}

Submit your manuscripts at

http://www.hindawi.com
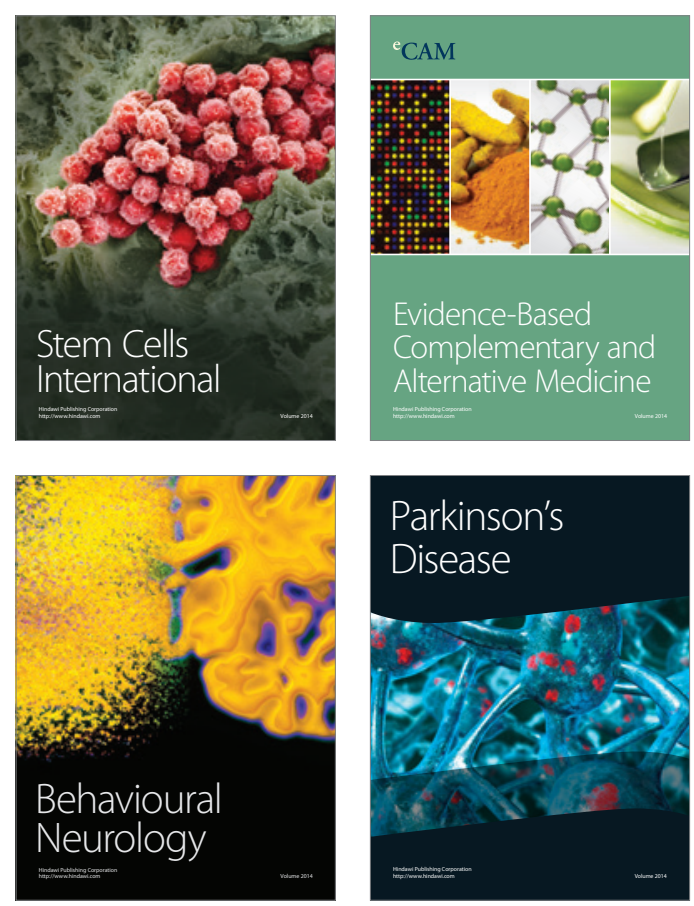

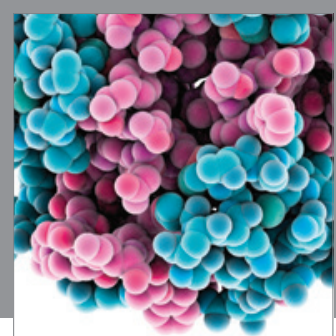

Journal of
Diabetes Research

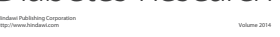

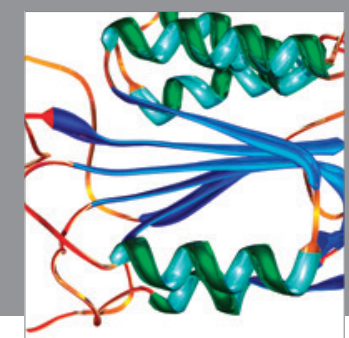

Disease Markers
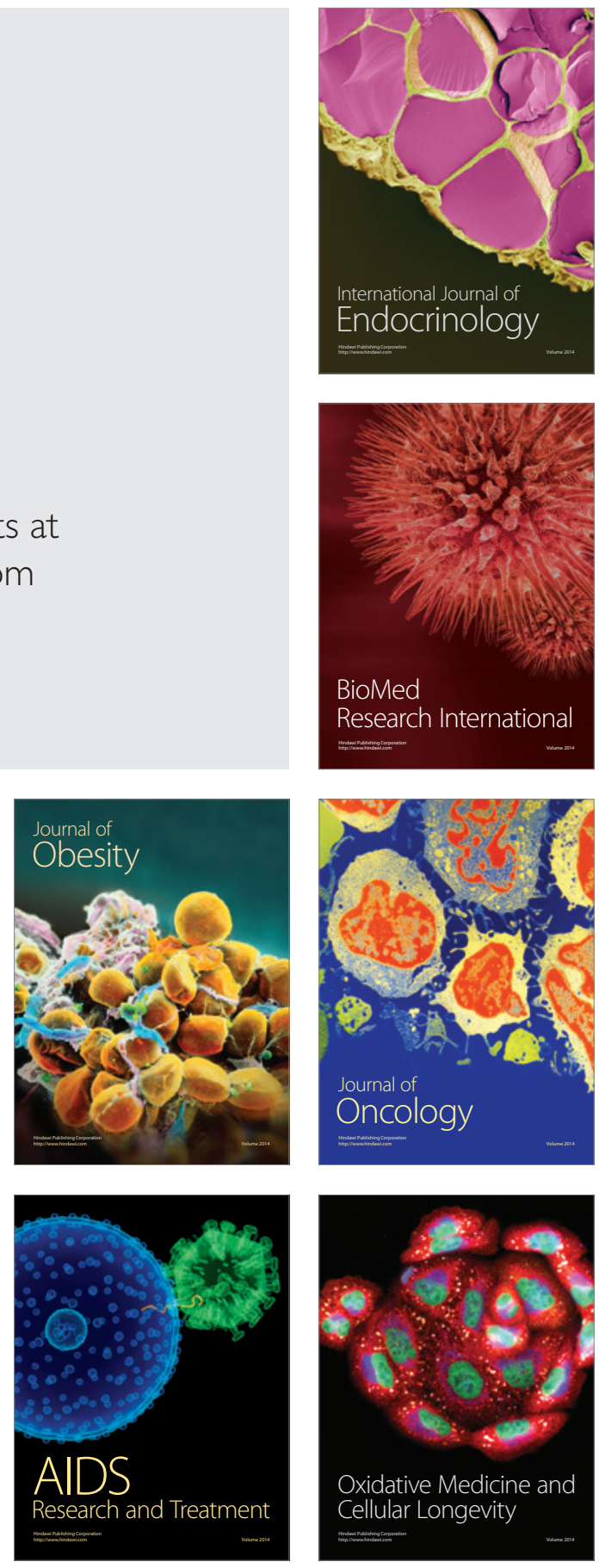